\title{
A Link State Dependent TDMA Protocol for Industrial Wireless Sensor Network Applications in Periodically Changing Environments
}

\author{
Valance Phua and Amitava Datta*
}

\begin{abstract}
Existing TDMA-based MAC protocols for wireless sensor networks are not specifically built to consider the harsh conditions of industrial environments where the communication channel is prone to signal fading. We describe the impact of periodically changing environment on small-scale fading effects in industrial indoor wireless networks. We use a site-specific ray tracer for predicting signal propagation based on a building blueprint representation to simulate the signal propagation paths between a receiver and transmitter in a periodically changing indoor environment. We show that the position of nodes and the periodic movements of objects with constant velocities in the environment have significant impact on signal fading, hence varying the resultant waveform perceived by a receiver. We present the results of two scenarios, each with different node placements and moving object velocities and we show that signal fading is approximately periodic. We then propose a TDMA-based MAC protocol for wireless sensor networks built for industrial applications that uses link state dependent scheduling. In our approach, nodes gather samples of the channel quality and generate prediction sets from the sample sets in independent slots. Using the prediction sets, nodes only wake up to transmit/receive during scheduled slots that are predicted to be clear and sleep during scheduled slots that may potentially cause a transmitted signal to fade. We simulate our proposed protocol and compare its performance with the well published Z-MAC protocol. We found that our protocol significantly improves packet throughput and energy consumption as compared to Z-MAC. We also found that in
\end{abstract}

*School of Computer Science \& Software Engineering, University of Western Australia, Perth, WA 6009, Australia. email:\{valance, datta \}@csse.uwa.edu.au 
conditions which are not perfect under our assumptions, the performance of our protocol degrades gracefully.

Index Terms - Wireless Sensor Network, Manufacturing, Fading, Multipath, Impulse Response, TDMA, MAC Protocol.

\section{Introduction}

Wireless communication in industrial applications has attracted much interest of late due to its low-cost setup and maintenance, flexibility, and easy configuration [21]. However, the harsh conditions of the wireless medium in most industrial environments pose a challenging task in designing a suitable protocol for wireless communication. With the presence of highly reflective surfaces and scattering objects in the environment, a transmitted signal goes through constant reflections, transmissions, and scattering, causing the energy of each multipath signal to dissipate in phase, amplitude, and time. Due to this effect of small-scale fading and multipath propagation of radio signals, the waveform received by a node is the result of multiple timeand spatially-dispersed copies of the transmitted signal and may distort or fade [14].

If we exclude indeterminate sources such as human movements, or movements of objects by factory operators, there is evidence that signal fading is approximately periodic $[13,10]$. The study performed in [13] provides evidence that the characteristic features of multipath fading is in fact a deterministic and spatial phenomenon. The authors have shown that in indoor environments, multipath fading is only determined by the topology of the surrounding environment, and is time-invariant in static conditions and is time-variant in dynamically changing environments. As a result, a periodic topology change in the environment results in a periodic and predictable pattern of multipath fading.

In this paper, we consider a fully automated industrial plant with objects or machine parts moving in periodic time intervals and show that periodic intervals of signal fading occur. When objects are in motion, all multipath components of a transmitted signal go through a timevarying Doppler shift as a result of path differences on each component [14]. Hence, the phase change on the resulting waveform received may or may not be distorted, depending upon the propagation path of the multipath components through the object locations at a specific snapshot of the environment at a particular time $t$. Due to the periodicity of the object motions, the object locations will be repeated after some time interval $\Delta t$ throughout the transmission cycle. 
The physical placements of sensor nodes in an industrial plant are very important because the magnitude of fading varies at different locations. It is difficult to estimate the severity of fading at every location of the industrial plant, as this involves monitoring the communication channel in the environment using complex and often, expensive equipments. Furthermore, when machineries in the plant are tuned to move at different speeds, the magnitude of fading at different locations in the environment changes. This would require a re-estimation of the severity of fading at every location in the industrial plant. It would also be unreasonable to keep moving the nodes until non-fading locations are found because in some situations, the sensor nodes are deployed in inaccessible locations, such as in gas chambers, radioactive enclosures, and other hazardous sites. In addition, it would also be expensive to shut down the plant just to relocate the sensor nodes. Hence, it is more sensible to design a network protocol that adapts to the environment.

Since the medium access control (MAC) layer is the core layer in the network protocol stack that determines the reliability and efficiency of data transmission, we propose a TDMAbased MAC protocol for WSNs specifically designed for industrial applications. There are many existing TDMA-based MAC protocols for WSN proposed such as in [15], [2] and [18] but to the best of our knowledge, none of them are specifically designed for industrial applications, where the conditions of the communication channel are harsher. Existing MAC protocols for WSN that have fault-tolerant capabilities such as [2] will try to repair communication links every time a distorted signal is encountered. If fading in the communication channel is periodic, such a protocol will spend most of its lifetime exchanging overhead packets to repair communication links rather than transmitting useful data. On the other hand, existing MAC protocols that do not have fault-tolerant capabilities such as [4] will not capture this fading phenomenon, thus increasing packet losses.

In our simulations, we compare the performance of the proposed MAC protocol with a widely published protocol, Z-MAC or Zebra-MAC [15]. Z-MAC is a hybrid MAC protocol that combines the advantages of both TDMA and CSMA, while minimizing their disadvantages. The underlying MAC scheme used by Z-MAC is CMSA, and it adopts TDMA during high contention periods, making it robust towards topology changes and time-varying channel conditions. In Z-MAC, TDMA time slots can be reused and non-owners of a slot can request to use the slot if it is not contended. The priority scheme adopted by Z-MAC for its slot policy makes the protocol tolerant to synchronization and slot assignment failures. Although Z-MAC is tolerant to clock synchronization and slot assignment failures, topology changes, and time- 
varying channel conditions, it is not built to account for the periodic multipath fading that occurs in indoor environments. However, since there is no known MAC protocol that is designed to account for this periodic fading in the environment, we take Z-MAC as a benchmark for comparison of our protocol. In addition, Z-MAC is also a good representation of a better protocol that has been shown to outperform several other existing MAC protocols [15].

Saleh et al [16] proposed a TDMA-based MAC protocol for general indoor wireless communications that incorporates a slow frequency hopping and coding technique. Each transmission in different frames within a superframe is done at a different frequency. The cycle repeats at each superframe. As most fading is continuous in a single frequency, this technique reduces the number of corrupted bits in a block of messages. Using the Reed-Solomon error-correcting algorithm, the block of messages is reconstructed at the receiver. However, this technique poses several problems in WSNs. Firstly, a transmitted signal requires a wide band of frequencies. This results in redundant frequencies at most times and wastes bandwidth. Secondly, a cyclical frequency hopping code needs to be preset at both transmitter and receiver nodes using a step frequency synthesizer. This is not available in the current hardware of most sensor nodes. Finally, it is expensive to run error-correcting codes in sensor nodes given the limited resources available.

Willig [20] proposed the use of a link state dependent scheduling with two-state Markov Model to counter the channel fading problem. In this approach, the channel quality is made up of two states, good and bad. Using transition properties of Markov chains [3], packets are only transmitted in good states. In our context, the Markov model is not suitable because the communication channel is assumed to be fading periodically and Rayleigh (random) fading is not applicable. Hence there is no continuous association between the two states, as assumed in the Markov model.

The rest of this paper is organized as follows. In section 2, we use a site-specific parallel ray-tracing simulator [19] to simulate the wireless communication in an industrial indoor environment with periodically moving objects. We show that under such environments, there is periodic distortion of signals due to multipath propagation. We also show that the patterns at which the signals distort repeats after a certain subset of time interval, hence creating a periodic cycle of successful transmission. We then propose a novel TDMA-based MAC protocol for wireless sensor networks specifically designed for industrial applications in section 3 . We incorporate a link state dependent scheduling (LSDS) approach for each independent slot, whereby a node only transmits/receives packet when a slot is predicted to be clear and delays transmis- 
sion/reception when a slot is predicted to be corrupted. We use a simple algorithm for observing the channel quality and computing the shortest repeats of the observation set. In section 4, we show by simulation that our proposed technique significantly improves packet delivery ratio and energy consumption as compared to the Z-MAC [15] protocol. We also show that in the presence of insufficient observation length, our protocol degrades gracefully and performs no worse than the Z-MAC protocol. Finally, we conclude this paper in section 5.

\section{Ray Tracing}

$S^{4} \mathrm{~W}[19,7]$ is a site-specific simulation tool that uses parallel ray tracing to simulate indoor wireless systems. It was developed at the Virginia Polytechnic Institute and State University. $\mathrm{S}^{4} \mathrm{~W}$ uses 3D geometrical optics ray tracing technique to approximate the impulse response received at a receiver node. In $\mathrm{S}^{4} \mathrm{~W}$, the power of each ray received is calculated using the model developed in [17] and given by equation 1 .

$$
P_{i}=P\left(d_{0}\right)-20 \log (d / \lambda)-n L_{r}-m L_{t}
$$

In equation $1, P_{i}$ is the power for the $i^{t h}$-ray, $P\left(d_{0}\right)$ is the transmitted power at reference distance $d_{0}, d$ is the total distance traveled by ray $i, \lambda$ is the wavelength of the ray, $n$ is the number of reflections, and $m$ is the number of transmissions. $L_{r}$ and $L_{t}$ are constants for the reflection and transmission losses. $\mathrm{S}^{4} \mathrm{~W}$ takes the standard AutoCAD's Drawing Interchange Format as its input and outputs a power delay profile that contains the impulse response of each ray received. The power delay profile includes the arrival time, power received, phase, and transmission and reception angles.

$\mathrm{S}^{4} \mathrm{~W}$ simulates signal propagation in the environment for a single transmission. Hence, a simulation of $\mathrm{S}^{4} \mathrm{~W}$ can be represented as the channel impulse response for a particular snapshot of time $t$. To simulate the fading channel as a series of time intervals, we take a series of snapshots in continuous time slices to represent the transmission of data between two nodes. The time interval, $\Delta t$, between two consecutive snapshots represents the data transmission cycle. To utilize $\mathrm{S}^{4} \mathrm{~W}$ to capture object motion in a periodic fashion, we assign a velocity, $v$, a direction of motion, a movement plane, and a pair of boundary points on the plane for each moving object in the environment. Using the basic principles of physics, we calculate the displacement of a moving object between time intervals by shifting its original position on the movement plane given by equation 2 . 


$$
d=v \cdot \Delta t
$$

The direction of shift is determined by the direction of motion of the object. If the object is moved outside the two boundary points on the movement plane, then the direction of motion for that object is negated.

Using the power delay profile obtained from running the simulations, we calculate the mean excess delay, $\bar{\tau}$ and RMS delay spread, $\sigma_{\tau}$, given by equations 3 and 4 respectively, where $\tau_{k}$ is the excess delay and $P\left(\tau_{k}\right)$ is the power received at $\tau_{k}$ [5].

$$
\begin{gathered}
\bar{\tau}=\frac{\sum_{k} P\left(\tau_{k}\right) \cdot \tau_{k}}{\sum_{k} P\left(\tau_{k}\right)} \\
\sigma_{\tau}=\sqrt{\frac{\sum_{k} P\left(\tau_{k}\right) \cdot\left(\tau_{k}-\bar{\tau}_{k}\right)^{2}}{\sum_{k} P\left(\tau_{k}\right)}}
\end{gathered}
$$

The mean excess delay and RMS delay spread characterize the time dispersion characteristics of the received signals and provide a good measure of multipath fading. Specifically, large RMS delay spread causes intersymbol interference, in which the signal is time dispersed. If the time dispersion of the signal exceeds the speed limit at which modulated pulses (i.e. the symbols) can be transmitted, the symbols will overlap and collide, and as a result distort the resulting waveform received. Hence, we use both mean excess delay and RMS delay spread to represent the state of the wireless channel in the industrial plant for each communication between two nodes.

\subsection{Ray Tracing Simulation Setup}

We use AutoCAD's Drawing Interchange Format to create a 3D representation of a manufacturing plant. The dimensions of the building is $50 \times 50 \times 12$ meters. The scene is made up of static objects, moving objects, external walls, internal walls, floor, roof, a transmitter, and a receiver. All objects are static except the moving objects. Using this scene setup, we simulated two different scenarios with different transmitter and receiver locations placed in the plant. We label each scenario as scenario 1 (SC1) and scenario 2 (SC2). Figures 1 and 2 are 3D representation of polyface meshes that describe SC1 and SC2 respectively. All objects in motion have constant random velocities associated with their movement and they are different for both scenarios. 


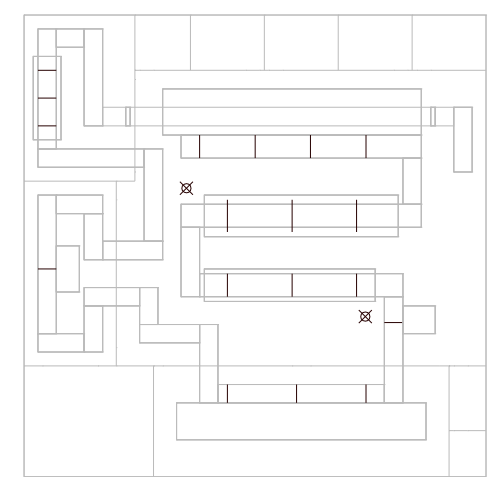

(a) Top view of the manufacturing plant.

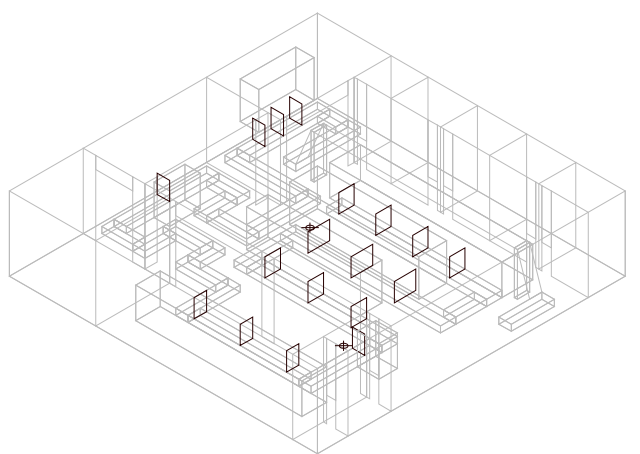

(b) $3 \mathrm{D}$ view of the the manufacturing plant.

Figure 1: Plant view of the manufacturing plant for SC1. Transmitter location is (11.94,-$7.65,0.00)$ and receiver location is $(-7.41,6.24,0.00)$. The bold rectangular planes represent moving panels with constant random velocities in the plant.

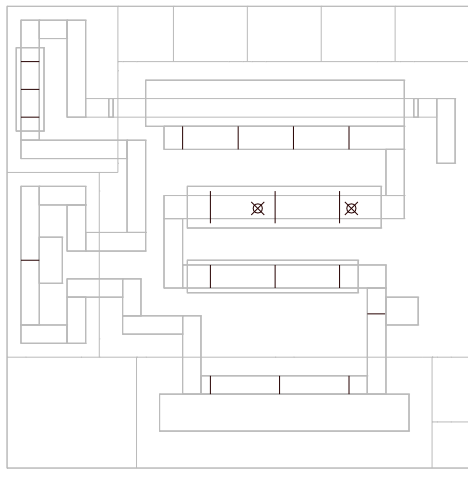

(a) Top view of the manufacturing plant.

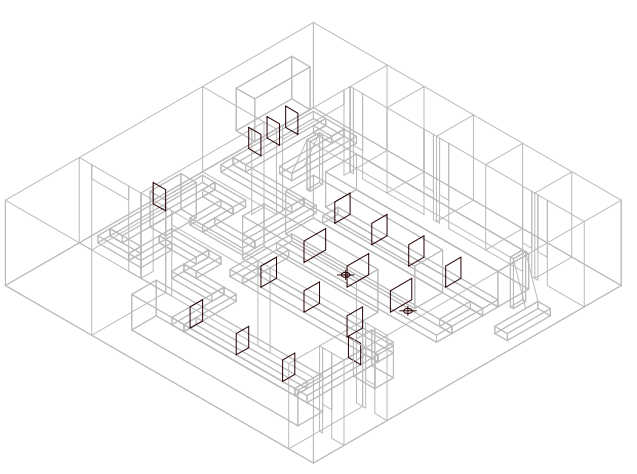

(b) $3 \mathrm{D}$ view of the the manufacturing plant.

Figure 2: Plant view of the manufacturing plant for SC2. Transmitter location is $(12.26,3.11,1.00)$ and receiver location is $(2.14,3.11,1.00)$. The bold rectangular planes represent moving panels with constant random velocities in the plant.

In the configuration file for $\mathrm{S}^{4} \mathrm{~W}$, we use the default specifications of Mica2 node by Crossbow Technology [8] for the operating frequency, transmission power, and receiver sensitivity. 
The operating frequency is set at $433 \mathrm{Mhz}$, transmission power at $-10 \mathrm{dBm}$, and minimum power at $-101 \mathrm{dBm}$. We set the path loss factor at 1 as this is common for indoor wireless propagation [14]. The reflection loss constant, $L_{r}$ from equation 1 is set at $2 \mathrm{~dB}$ loss for every ray intersection. This value is used because objects in most manufacturing plants are made of metal and have very high reflection coefficients. Since most of the energy from an incident ray is reflected back on a metal surface, we specify a lower-than-average value of $L_{r}$. In our simulations, we ignore ray transmissions through objects in the scene and set the transmission loss constant, $L_{t}$ to a large value. This is because, we assume that there is very little transmission of radio signals through objects in most manufacturing plants and due to limitations of $\mathrm{S}^{4} \mathrm{~W}$, we cannot specify individual transmission coefficients on each object surface. Our chosen values of $L_{r}$ and $L_{t}$ are reasonable since we are interested in amplifying the effects of reflection in the manufacturing plant.

We simulate each scenario for a logical run time of 2000 seconds, which is sufficient to observe the periodic cycle of signal distortions between time intervals. The transmission rate is set at 1 transmission per second. For each transmission, all components of the transmitted signal, along with the multipath components are ray traced through the scene and each component of the multipath signal received is reflected in the power delay profile. Throughout the run time, all moving objects are in continuous motion with velocities, $v$ set at $0.1 \mathrm{~m} / \mathrm{s}<v<2.0 \mathrm{~m} / \mathrm{s}$, and plane boundary, $b$ set at $2 m<b<3 m$ on the corresponding plane of movement.

\subsection{Ray Tracing Results \& Discussion}

Figure 3 shows the power delay profile for SC1 and SC2 as a function of time, excess delay, and magnitude of received power. The impulse response for each reception is represented by the excess delay and magnitude of received power axes. The time axis represents a series of impulse responses for receptions at different time intervals. Figure 3(a) shows the magnitude of the received power in SC1 fluctuates intermittently in the excess delay range of approximately $250 \mathrm{~ns}$ to $700 \mathrm{~ns}$. During this period, each crest that arrives after a trough represents an amplified multipath component that arrives at a later time. If the magnitude of this signal fluctuation is large, the received symbols may overlap causing intersymbol interference that would lead to distortion of the signal wavefront received. At different instances of time, the received impulses have different variations in magnitude of fluctuation. However, this variation in impulse response forms a repetitive subset after a few transmissions, which can be explained by the toothed edges between the crest and trough of Figures 3(a) and 3(b) after a certain time period. 
The periodic subsets of randomly fluctuating impulses in SC1 is caused by the periodic motion of objects in the manufacturing plant. We can think of this as getting a snapshot of the environment during an instance of time at $t_{0}$. At $t_{0}$, all objects in the environment are aligned at particular positions, $P$. Since all moving objects have constant velocities of motion, they will eventually align in the same position $P$ after a certain time period of $\Delta t$. Hence the impulse response within $\Delta t$ is sporadic, but repeats when objects are realigned in the environment after $\Delta t$. Each periodic subset of the toothed edges between the crest and trough can be represented by $\left(t_{i}+\Delta t\right)$, where $t_{i}$ is the time interval between the repetitive subsets.

Figure 3(b) show the impulse response of SC2. The power received gradually decreases, with very little fluctuations in the excess delay axis for each transmission interval. This suggests that there is very small or no intersymbol interference due to multipath component arrivals and would not likely cause the received signal to fade. Due to the small distance between the transmitter and the receiver, the small variation in the magnitude of received impulses between time intervals would unlikely cause intermittent fading periods. Figures 4 and 6 are the expanded versions of Figure 3. The parameters of magnitude of power received and excess delay are fixed in Figures 4 and 6 respectively.

The mean excess delay reflects on the mean delay of signals from the arrival of the first signal and the RMS delay spread is the square root of the second central moment of the received power [6]. Both mean excess delay and RMS delay spread are primarily affected by the path distance between the transmitter and receiver and the delay of multipath components that arrive at the receiver. In our simulation, both transmitters and receivers are static. Hence, the effect of path distance between the two nodes on the variation of mean excess delay and RMS delay spread at different time intervals is negligible, and is solely affected by the delay of multipath components that arrive at the receiver. Therefore, a large magnitude of difference in mean excess delay and RMS delay spread suggests a higher probability of signal fading.

Figures 4 and 5 show the mean excess delays and RMS Delay spreads of SC1 and SC2. In Figures 4(a) and 5(a), the fluctuations in magnitude of mean excess delay and RMS delay spread are large and this suggests that the received signals are fading at certain time intervals. Although these fluctuations are rapid in SC2 (from Figures 4(b) and 5(b)), the magnitudes of variation are small and would probably result in very small intersymbol interference that can be ignored. We also note that when the transmitter and receiver are placed in close distance to each other, such as in SC2, fading is less apparent as compared to SC1, where the transmitter and receiver are placed further apart. 


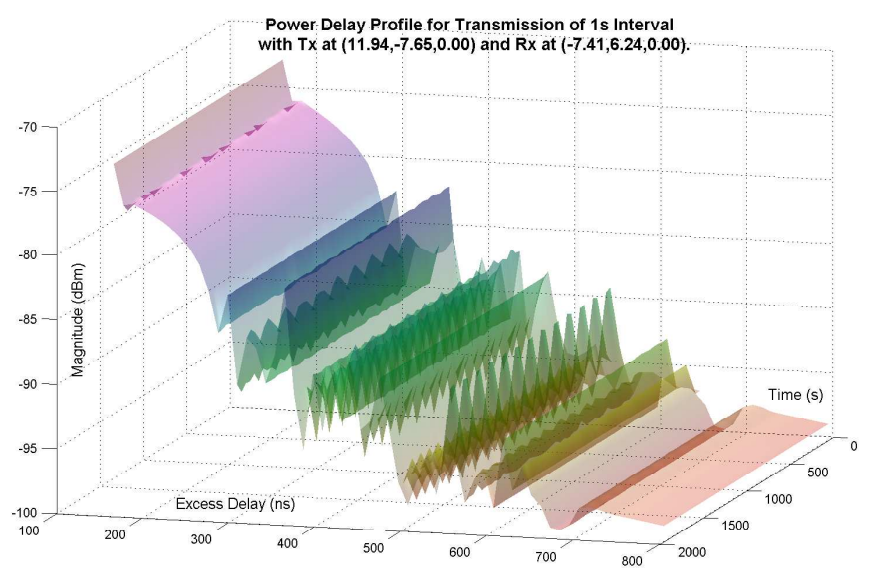

(a) SC1

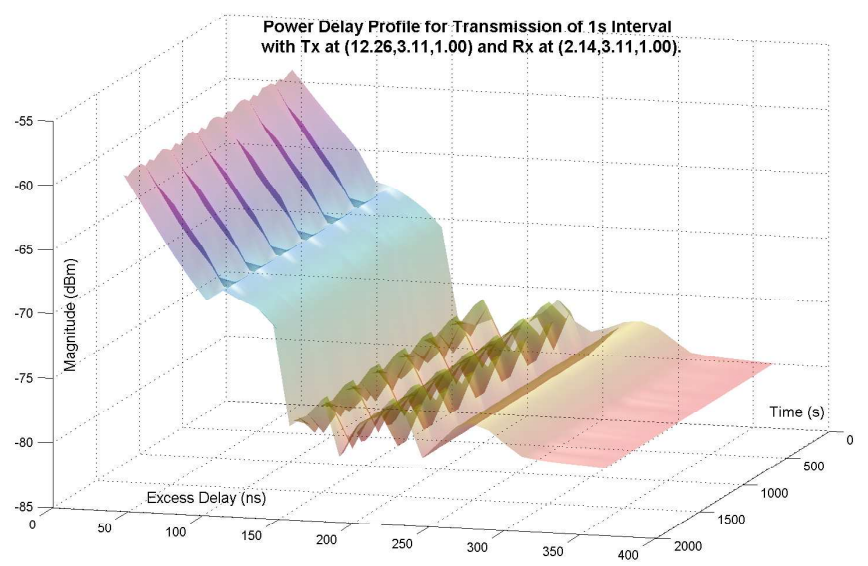

(b) $\mathrm{SC} 2$

Figure 3: The power delay profile for $\mathrm{SC} 1$ and $\mathrm{SC} 2$ as a function of time, excess delay, and magnitude of received power.

Figure 6 shows the normalized power received by the receiver as a function of time. In SC1 (Figure 6(a)), we see that the normalized power received randomly fluctuates between the range of approximately $-88.8 \mathrm{dBm}$ and $-91.4 \mathrm{dBm}$. In SC2 (Figure 6(b)), the normalized power received fluctuates between the range of approximately $-73.8 \mathrm{dBm}$ and $-75.1 \mathrm{dBm}$. For both 


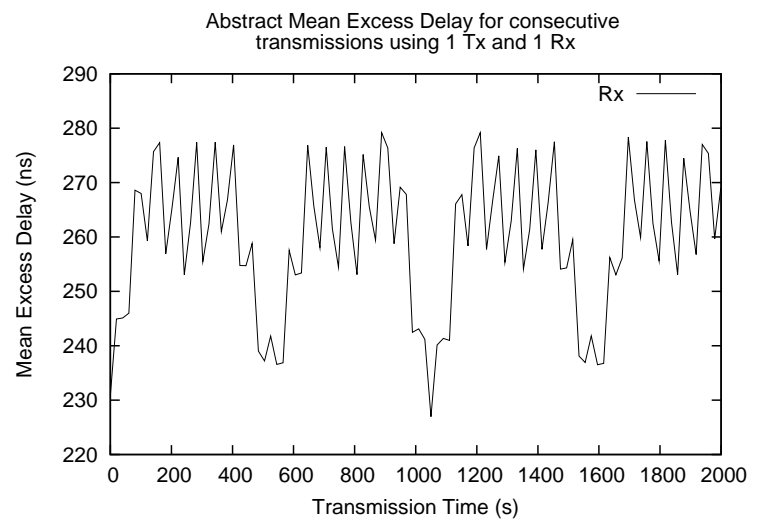

(a) SC1

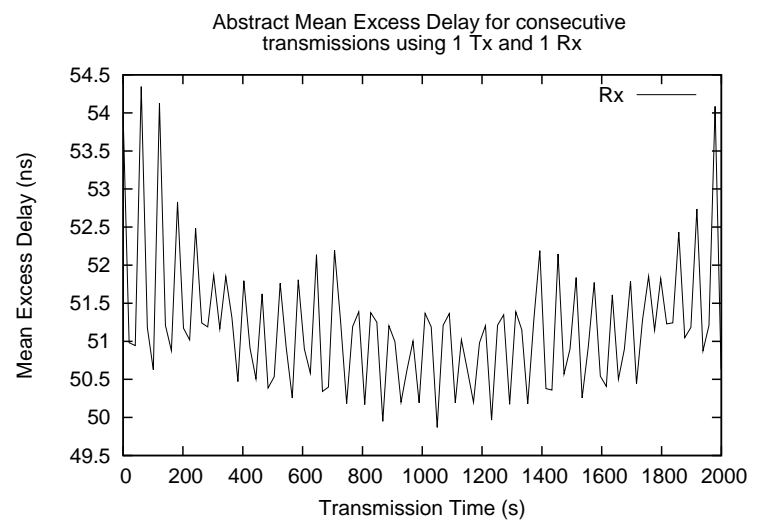

(b) SC2

Figure 4: The mean excess delay for SC1 and SC2.

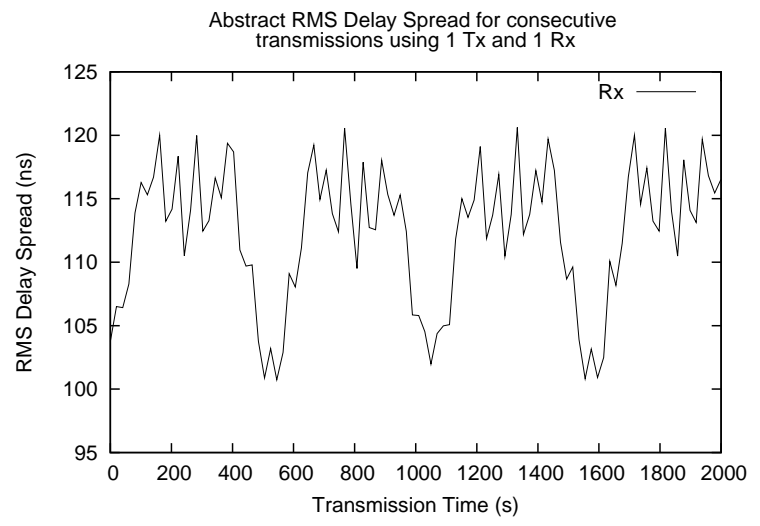

(a) SC1

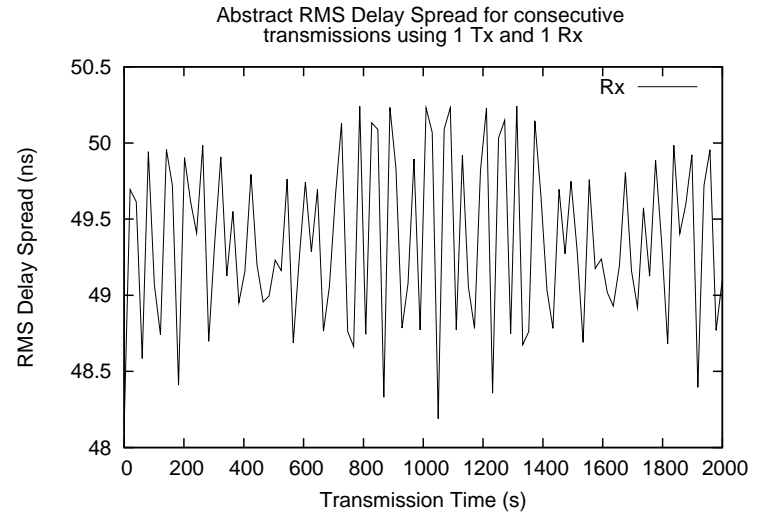

(b) $\mathrm{SC} 2$

Figure 5: The RMS delay spread for SC1 and SC2.

SC1 and SC2, we also observe that the fluctuation pattern of the normalized power received from 0 seconds to approximately 1100 seconds is repeated at 1100s. From the normalized power received, we can perceive signal fading as a periodic event. Our simulation results agree with the findings in [13].

For a sensor node, the receiver power threshold, $R$, represents the limit at which acceptable bit-error-rate performance of the receiver is expected. The value of $R$ depends on the receiver 


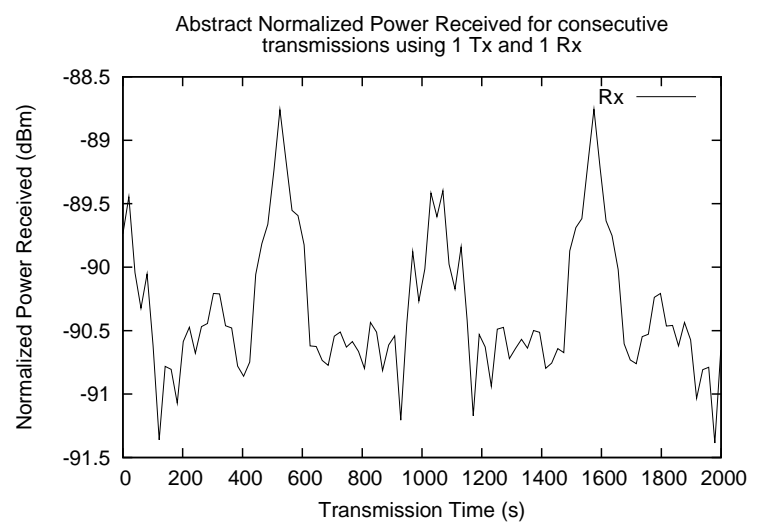

(a) SC1

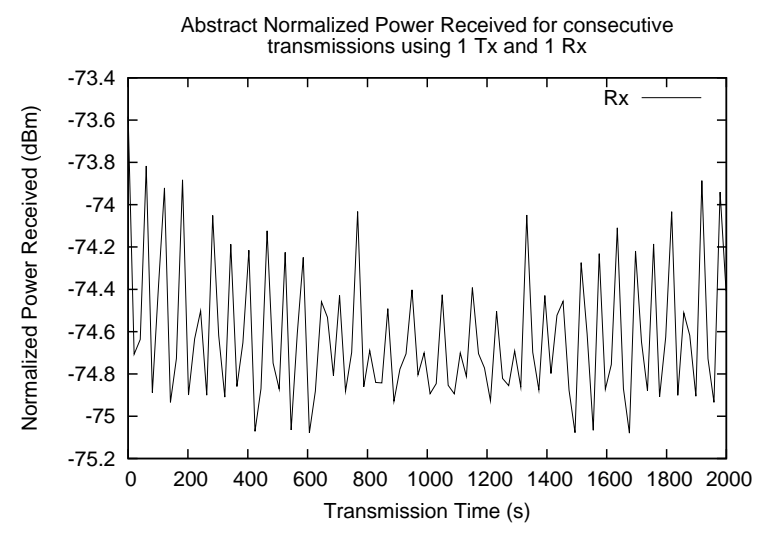

(b) SC2

Figure 6: The normalized power received for SC1 and SC2.

sensitivity and acceptable signal-to-noise ratio of individual hardware. We give an example of this periodic signal fading for SC1 in Figure 7. For illustration purpose, we set $R$ at $-90 \mathrm{dBm}$ such that any received signal with power values that fall below $R$ is unacceptable. The grey areas are periods when transmitted signals go through fading. We see that in a fully automated plant with periodically moving objects, signal fading eventually converges to a periodic burst of fading intervals throughout the transmission cycle, which will result in packet losses.

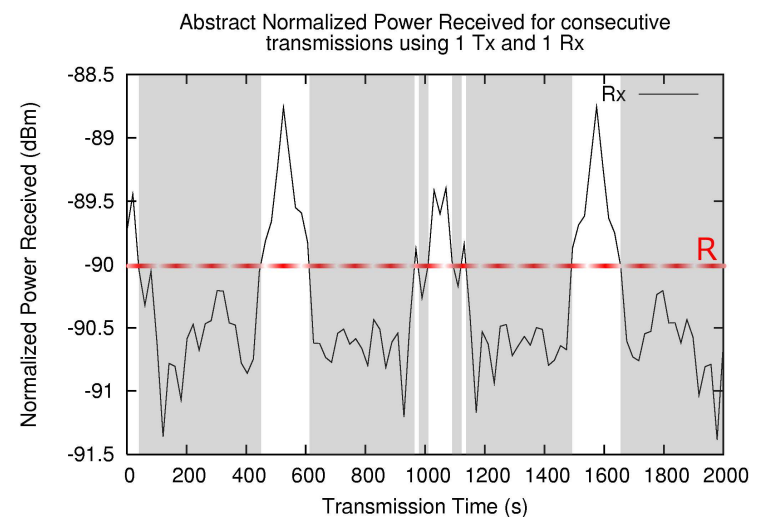

Figure 7: Periodic signal fading in SC1 with received power threshold, $R$ set at $-90 \mathrm{dBm}$. The grey areas are periods at which signal is distorted due to multipath propagation. 


\section{Link State Dependent Scheduling}

When sensor nodes are deployed in manufacturing plants, it is difficult to estimate the degree of multipath fading between a transmitter and a receiver at different locations. At some transmitterreceiver placements, fading may be severe and at some other placements, there may not be any fading. As such, instead of physically moving the sensor nodes until non-fading locations are found, it would be more effective to develop a protocol that adapts to the environment. Having identified that signal fading in an industrial plant lead to periodic packet losses, we propose a MAC protocol that is able to operate efficiently in this environment. Our proposed MAC protocol is a TDMA-based MAC protocol that uses link state dependent scheduling (LSDS) to adapt to the periodicity of the signal fading periods locally in each TDMA time slot. Each node avoids transmission during slots that are predicted to be affected by the fading channel. We use the terminology of good and bad for time slots that are predicted to be clear of or affected by signal fading in the communication channel respectively.

The protocol initially starts in a random-access mode. During this startup phase, tokens and TDMA slot assignments for unique communication between nodes (TDMA schedules) are exchanged between all nodes in the network. Once all nodes have discovered their neighbours and exchanged schedules, they communicate in TDMA mode. When the nodes initially switch to the TDMA mode of communication, they gather training set data for a specified length of time, $t$, for each slot to build the prediction set for subsequent transmissions and receptions. After $t$, the nodes operate in a signal fading aware (SF-Aware) mode, where the prediction set built for each slot is utilized to determine if transmission/reception should take place during a particular scheduled slot.

\subsection{TDMA Frame Format}

Figure 8 shows the communication channel, which can be thought of as being divided into a series of TDMA time frames. A TDMA frame consists of communication slots and a control slot. Each communication slot is unique for communication between a pair of sensor nodes and no data collision is possible in this slot. From a sensor node's point of view, the stream of communication in a slot is either uplink or downlink for transmit and receive respectively. A node turns its radio on and listens for packet during its downlink slot. Conversely, a node turns its radio on and transmits data during its uplink slot. The downlink slot of a parent-to-child link 


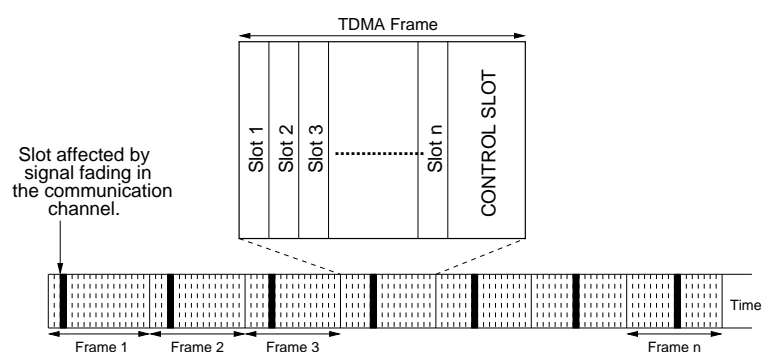

Figure 8: TDMA frame format of the proposed protocol. The shaded slot in each frame represents a slot that is affected by the periodic signal fading in the communication channel.

is used for clock synchronization. The control slot at the end of each frame is a contentionbased slot used for future development of the protocol to support the addition of new nodes. Any slot in a frame can be potentially affected by the periodic signal fading in the channel. The sizes of each frame, communication slot, and control slot is fixed.

\subsection{Startup Phase}

During the startup phase, all nodes in the network discover their neighbours and establish TDMA schedules in random-access mode. In our protocol, we use a bottom-up tree-based token passing approach, where each relay node knows the exact number of descendants for each of its children. The base station and relay nodes assign TDMA schedules by allocating the exact number of slots needed to support each child and its descendants for exclusive communication. The nodes then exchange their TDMA schedules with neighbouring nodes and communicate in TDMA mode.

Since token passing and TDMA schedule assignment are widely known approaches $[9,11$, 4], we will omit the detailed discussion on these steps. Full discussion of our startup phase can be found in [12]. During schedule passing, the assignments of slots start from the root of the tree, and propagate through nodes at each level of the tree. The branch count of each child is the number of uplink slots a parent would assign for that child. In addition, a downlink slot is also assigned for each child to support parent-to-child communication. 


\subsection{Observation Phase}

In the observation phase, sensor nodes learn the periodic pattern of the length and interval of the fading signal in the channel. The length of a fade is the duration that a fading signal lasts and the interval of a fade is the frequency of its occurrence. During this observation phase, all sensor nodes transmit and receive data during their scheduled slots, which they have negotiated during the startup phase. However, these slots may be affected by signal fading in the communication channel and are labeled as bad slots if transmissions/receptions fail or good slots if transmissions/receptions are successful. All patterns of good and bad states for each independent slot are buffered in a node's flash memory and are used as a training set to establish a prediction set that is the shortest repeat of the training set. In our approach, it is only possible for a receiver to gather the training set and build a prediction set because since it is receiving, it can detect corrupted data or packet not received during a scheduled receive slot. Hence the receiver establishes the prediction set and exchanges the list with the sending node for that slot during an uplink slot at the beginning of the SF-Aware phase, as explained further in section 3.4. If the prediction set is large, then it can be fragmented into multiple packets and sent in simultaneous frame cycles.

Our approach works as follows. A node maintains a pair of counters, $c$ and $d$, both initialized to zero for each receive slot in its schedule. $c$ and $d$ are the number of simultaneous occurrences of good and bad states in a particular slot of a frame. For a scheduled receive slot $r$, a node increments $c$ by one if it successfully receives a packet from the corresponding sender node. It keeps incrementing $c$ by one for each successful reception in contiguous frame cycles at slot $r$ until it encounters slot $r$ with erroneous or no reception, where it increments $d$ by one. For every consecutive bad slot in subsequent frame cycles for slot $r$, the node increments $d$ by one until it encounters the next successful reception from its corresponding node. When this next successful reception is encountered, the node appends $(c, d)$ in the sample set $S$ for slot $r$ and resets $(c, d)$ to $(1,0)$. The node repeats this process in slot $r$ throughout the observation phase. The first tuple observed is ignored because this pair may start in the middle of a series of good or bad slots during the transition from startup phase to the observation phase. All sample sets mentioned in the following discussion do not include the first tuple observed.

Let the collected sample set be $S=<s_{0}, s_{1}, \ldots, s_{j}>$ where each $s_{i}, i=0 \ldots j$, is a tuple $(c, d)_{i}$. Each $s_{i}$ observed is added to the set $S$ and a prediction set $P=<p_{0}, p_{1}, \ldots, p_{k}>, k \leq j$, is computed from $S$ as follows: For each $s_{i}$ in $S$ and for each $p_{i}$ in $P$ starting from $i=0 \ldots j$, if $P$ is empty or $s_{i} \neq p_{i \bmod (k+1)}$, then we add $s_{k+1}, s_{k+2}, \ldots s_{i}, k \leq i$, in $P$ and repeat from $i=0$. 
This is because, there is no known prediction pattern if $\mathrm{P}$ is empty and the periodicity of $\mathrm{S}$ in $\mathrm{P}$ no longer holds if $s_{i} \neq p_{i \bmod (k+1)}$. Given $P$, if $k<j$, then $P$ is the shortest representation of $S$ where each $P$ is a simultaneous repeat of a subset of $S$. If $k=j$ at the end of the observation phase, then $S=P$ and there is no known periodic occurrence in $S$. Signal fading in the channel is thus random.

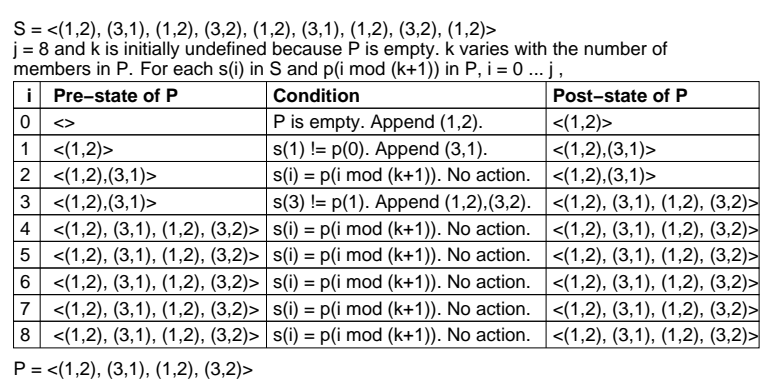

Figure 9: An example of computing the prediction set $P$ from the sample set $S$. $P$ is the shortest repeat of $S$.

To illustrate our algorithm, we give a simple example of computing the prediction set $P$ from a sample set $S$ in Figure 9. At $i=0, P$ is empty, so we append $s_{0}=(1,2)$ to $P$. At $i=1, s_{1}=(3,1) \neq p_{1 \bmod 1}=p_{0}=(1,2)$, hence we append $s_{1}=(3,1)$ to $P$. At $i=2$, $s_{0}=p_{0}, s_{1}=p_{1}$, and $s_{2}=p_{0}$, hence no action is taken. At $i=3, s_{3}=(3,2) \neq p_{3 \bmod 2}=$ $p_{1}=(3,1)$, hence we append $s_{2}=(1,2)$ and $s_{3}=(3,2)$ to $P$. For $i=4 \ldots 8, s_{i}=p_{i \bmod (k+1)}$, hence no action is taken. The resulting prediction set $P$ that we obtain from $S$ is therefore, $P=<(1,2),(3,1),(1,2),(3,2)>$. $P$ is the shortest repeat for $S$. The visual representation of the our TDMA schedule utilizing the prediction set $P$ can be seen in Figure 10, where the series of pairs in $P$ represents the good and bad transmit/receive slots of simultaneous TDMA frames.

\subsubsection{Observation Length}

The time taken for a node to be in the observation phase is the observation length. If the observation length is too short, the periodicity of the signal fading may not be completely captured and this would cause the prediction set $P$ to reflect a sporadic fading channel. If the observation length is too long, then there will be redundant repetitions in the sample set and memory resource may be insufficient. Hence it is important that an ideal observation length is chosen. 


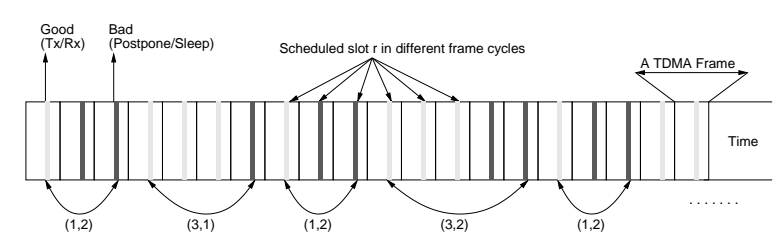

Figure 10: Channel representation of the use of prediction set for a slot $r$ in the TDMA schedule. Nodes transmit/receive when slot $r$ is good and postpone transmission/reception when slot $r$ is bad. Both transmitter and recipient nodes have the prediction set $P=<$ $(1,2),(3,1),(1,2),(3,2)>$.

The ideal observation length is preset at all nodes in the network before deployment.

Before we present the method of determining the ideal observation length, we assume that the interval of the obstruction source that causes the signal to fade (obstruction interval) is known in advance. If there are multiple obstruction intervals, then we assume the source with the longest obstruction interval is known in advance. We associate the obstruction interval with movements of machineries in the industrial site. Since the periodic movement of metal parts causes periodic signal fading, we take the obstruction interval to be the time interval at which a machine repeats its movement. We first present the parameters that contribute to the determination of the ideal observation length, namely:

\section{- Length of a frame - $F$}

\section{- Number of slots in a frame - $N$}

\section{- Frequency of reception - $T$}

\section{- (Longest) Obstruction interval - $O$}

Given these parameters, we find the most conservative estimate of the ideal observation length, $O_{t}$. In the worst case, we can guarantee that signal fading pattern at a particular slot would re-occur in that same slot after it has cycled through all the slots in subsequent frames. The time required for a node to observe two consecutive patterns depends on how frequent a node receives a packet in a slot (i.e. receive a packet in every $T$ seconds). Hence, the maximum observation time required to capture the entire cycle of a state is the product of the length of a frame, number of slots in a frame, frequency of reception, and obstruction interval. Since there are two states in our protocol, the ideal observation length required is given by equation 5 . 


$$
O_{t}=2 N F T O
$$

\subsection{SF-Aware Phase}

When nodes first enter the SF-Aware phase, they transmit all prediction sets (fragmented if necessary) of their downlink slots to the corresponding nodes of the affected slots in their respective uplink slots. During this exchange of prediction sets, the nodes append all $c$ and $d$ counters and the pointers to the current tuple in the prediction sets of each slot. To save memory resource, all sample sets are deleted once they are no longer required. When prediction sets are transferred, normal transmissions of sensed data are suspended and resumed only when all prediction sets have been sent.

When a node receives a complete prediction set from a corresponding node for slot $r$, it uses the prediction set in subsequent transmissions during slot $r$ to change the mode of scheduling from round-robin to link state dependent. Since the corresponding receiver has the same copy of the prediction set, both nodes can synchronize their wake up and postpone periods during slot $r$ at every frame cycle. This is done as follows. During a particular downlink slot for A and uplink slot for $\mathrm{B}$, node $\mathrm{A}$ receives a complete prediction set $P=<p_{0}, p_{1}, \ldots, p_{k}>$, along with the $c$ and $d$ counters, and a pointer $i$ to the current tuple in $P$, from node B for slot $r$. A stores this information in its neighbour list. In order to determine if both nodes A and B should wake up or sleep in the next slot $r$, they both check with $P, i, c$ and $d$ counters in the following manner:

For tuple $s_{i}, i=0 \ldots k$, in $P$, where $s_{i}=\left(c^{\prime}, d^{\prime}\right)_{i}$,

- If $c<c^{\prime}$, then increment $c$ by one and transmit/receive.

- If $c=c^{\prime}$ and $d<d^{\prime}$, then increment $d$ by one and postpone.

- If $c=c^{\prime}$ and $d=d^{\prime}$, then set $c=1, d=0$, and $i=i \bmod (k+1)$ and transmit/receive.

In the algorithm above, $c^{\prime}$ and $d^{\prime}$ in $\left(c^{\prime}, d^{\prime}\right)_{i}$ are the values of the tuple in prediction set $P$ at position $s_{i} . c$ and $d$ are the $(c, d)$ counters in section 3.3 that were sent from node $\mathrm{B}$ to $\mathrm{A}$.

After long periods of time, the actual cycle of the physically occurring fades may drift from the predicted cycle of fades in prediction set $P$. As a result, this may cause a harmonic drift between the actual fading cycle and the predicted cycle. To prevent this from happening, the 
base station periodically initiates requests to all nodes in the network to switch their modes back to the observation phase after a long period of time.

\section{Simulation Results}

We verify the performance of our proposed protocol by simulating the protocol using the discrete-event network simulator, ns-2 [1]. For comparison, we also simulated a hybrid (TDMA and CSMA) MAC protocol for wireless sensor networks, Z-MAC [15], in ns-2. In our simulations, 50 nodes are randomly deployed in a 500x500 square meter area and each deployment forms a connected network. The transmission range of each node is fixed at 100 meters. We use a single source of obstruction that causes signals to fade in the channel and simulate both protocols in fixed combinations of the obstruction lengths, obstruction intervals, and ideal observation times of each node with values as the following:

- Obstruction lengths - 4.1, 5.2, 6.3, and 7.4 seconds.

- Obstruction Intervals - 0.1, 0.7, 1.3, 1.9, and 2.5 seconds.

- Observation times - Steps of $\frac{1}{5}^{\text {th }}$ of the ideal observation time.

We simulate each scenario for a logical runtime of 172800 seconds ( 2 days). The data transmission rate for both protocols is set at 1.28 seconds. To show that our proposed protocol is tolerant to infrequent random fading, we introduce sporadic obstructions at random intervals between 500 and 1000 seconds that last for a random period of between 0.1 and 0.5 seconds for both protocols. In addition, we set the Z-MAC protocol to run in a dynamic contention level mode, and simulate Z-MAC with and without acknowledgment transmissions.

Figure 11 shows the node-to-node packet delivery ratio of both protocols with respect to the observation ratio used in the proposed protocol. We take the observation ratio to be actual observation length used against the ideal observation length. As expected, our proposed protocol significantly improves packet delivery ratio compared to Z-MAC in both with and without acknowledgment transmissions, provided the ideal observation length is used. When the ideal observation length is used, the packet delivery ratio of our proposed protocol is almost $100 \%$ for all cases, even with the presence of sporadic fading at very infrequent intervals. However when insufficient observation length is used, the proposed protocol does not optimize packet 


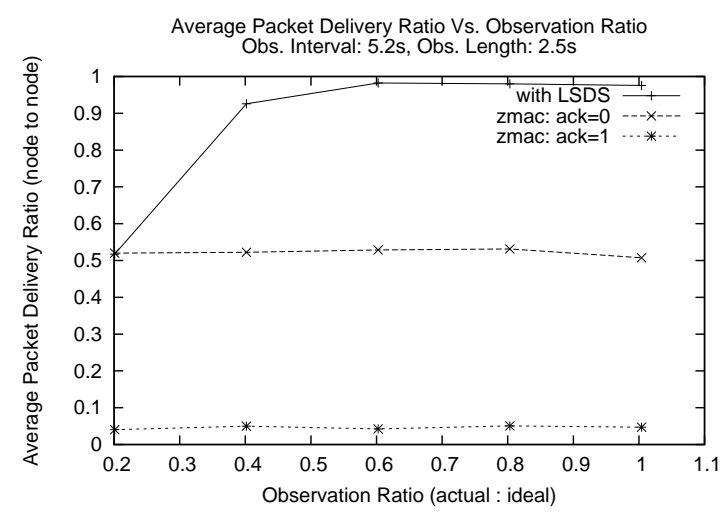

Figure 11: Packet Delivery Ratio vs. Observation Ratio (actual:ideal) for obstruction interval of $5.2 \mathrm{~s}$ and obstruction length of $2.5 \mathrm{~s}$.

delivery ratio and the performance of our protocol is about the same with Z-MAC without acknowledgement transmissions. Although the ideal observation length guarantees the protocol captures the entire cycle of periodic signal fading in the channel, we see that in most situations, the observation length required is less than the ideal observation length for packet delivery ratio to reach its maximum.

From our results, we also note that the performance of Z-MAC using acknowledgment transmissions achieves a much lower packet delivery ratio as compared to our proposed protocol and Z-MAC without acknowledgment transmissions. This is because, the acknowledgment packets sent by a receiver node may be affected by multipath fading in the environment, therefore requiring retransmissions of these acknowledgment packets. Furthermore, the retransmitted acknowledgments may also be affected by multipath fading, requiring further retransmissions of the acknowledgment packets. Hence using Z-MAC with acknowledgment transmissions is not ideal in environments where multipath fading is intense.

Figures 12 and 13 show the node-to-node packet delivery ratio of both simulated protocols with respect to obstruction frequency ratio. The obstruction frequency ratio is the ratio between the obstruction length and the obstruction interval, that is the period at which a fade lasts when it occurs at every interval. We use the obstruction frequency ratio as a unit of measurement because it gives a good description on the impact of the average packet delivery ratio at the 


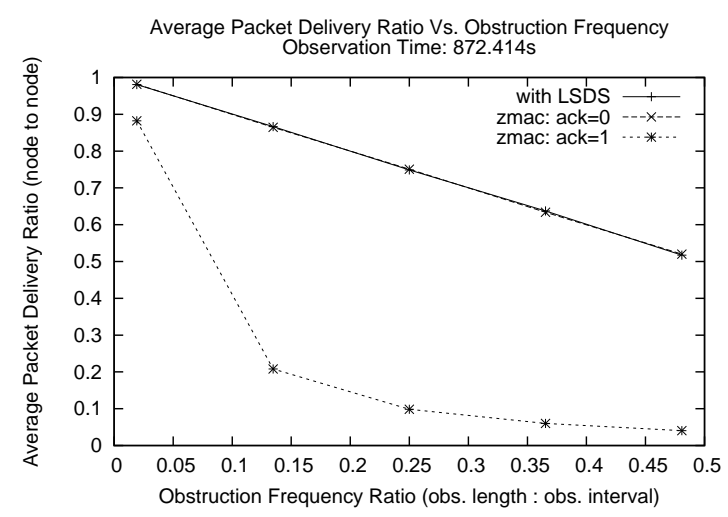

Figure 12: Packet Delivery Ratio vs. \% Obstruction Frequency Ratio (length:interval) for obstruction interval of $5.2 \mathrm{~s}$ and observation length of $872.414 \mathrm{~s}$.

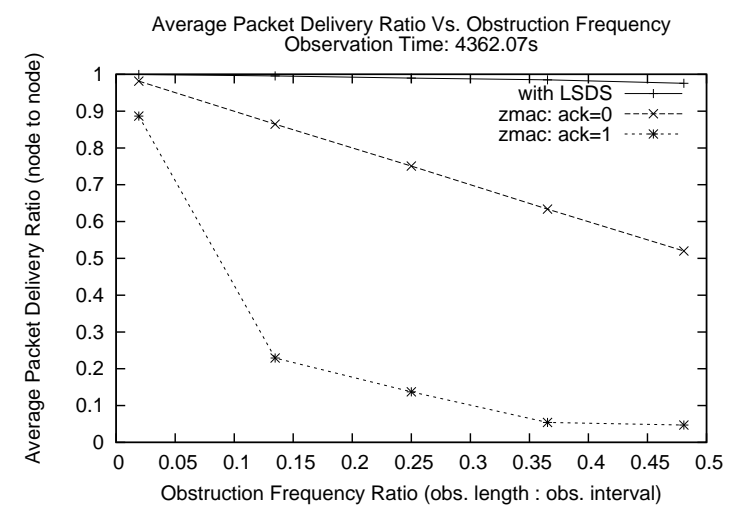

Figure 13: Packet Delivery Ratio vs. \% Obstruction Frequency Ratio (length:interval) for obstruction interval of $5.2 \mathrm{~s}$ and observation length of $4362.070 \mathrm{~s}$.

different intensity and regularity of fading.

From the results, we see that packet delivery ratio in the proposed protocol is also significantly higher than Z-MAC with or without acknowledgment transmissions, provided the observation length is sufficient. From Figure 12, the average packet delivery ratios for both the proposed protocol (with LSDS) and Z-MAC without acknowledgment retransmissions (zmac: ack=0) are almost overlapping for all values of obstruction frequency ratios. This suggests that the average packet delivery ratio is almost the same for the proposed protocol and Z-MAC without acknowledgment transmissions if the observation length used is small and insufficient. Hence, even if the prediction set is erroneous as a result from the insufficient observation length, 


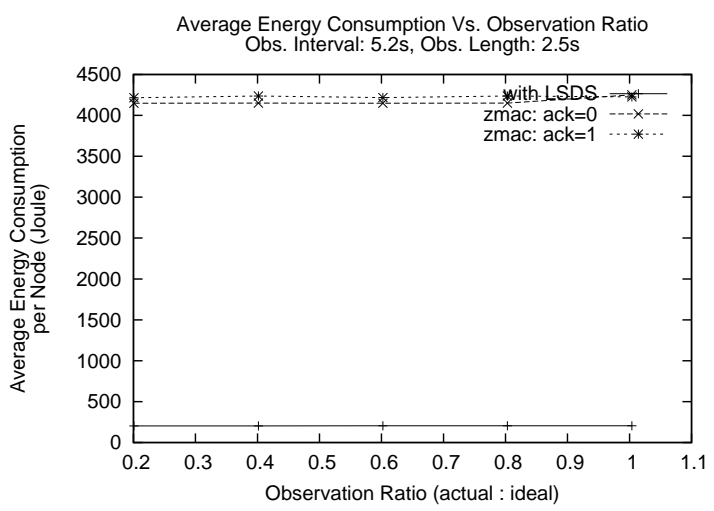

Figure 14: Average Energy Consumption vs. Observation Ratio (actual:ideal) for obstruction interval of $5.2 \mathrm{~s}$, obstruction length of $2.5 \mathrm{~s}$.

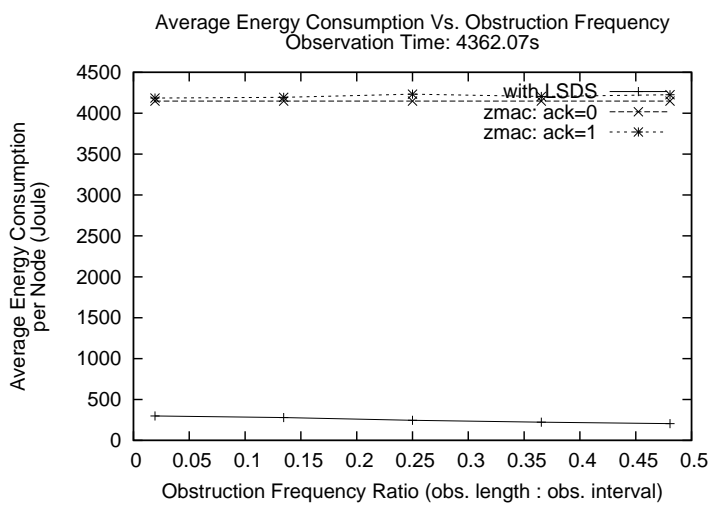

Figure 15: Average Energy Consumption vs. \% Obstruction Frequency Ratio (length:interval) for obstruction interval of 5.2s and observation length of $4362.070 \mathrm{~s}$.

the proposed approach performs no worse than Z-MAC. However when sufficient observation length is provided, we see that the packet delivery ratio of our proposed protocol decreases at a much lower rate and still maintains close to $100 \%$ performance compared to Z-MAC as the obstruction frequency ratio increases (Figure 13). This is because when the obstruction frequency ratio increases, the chances of more packets being corrupted or lost in the channel also increases. On the contrary, if we postpone transmission during bad slots and only transmit during good slots, this ensures a maximum packet delivery ratio regardless of the obstruction frequency ratio. 
Figures 14 and 15 show the average energy consumed by a node throughout the simulation time. We see that our proposed protocol consumes less energy as compared to Z-MAC with and without acknowledgment transmissions. Our protocol uses less energy because it operates in a passive mode. In Z-MAC, transmission/reception takes place during every scheduled slot regardless of the channel quality. Hence, energy is wasted when nodes transmit/receive during bad slots. In our proposed protocol, energy is conserved by avoiding transmission/reception and sleeping during postpone periods.

Our results also indicate that our proposed protocol suffers higher latency in one-hop nodeto-node and overall node-to-base packet delivery as compared to Z-MAC with and without acknowledgment transmissions. This is because our protocol buffers packets as it postpones transmissions/receptions during bad slots. Although Z-MAC have minimal one-hop node-tonode and overall node-to-base latency in packet delivery, the majority of the transmitted packets are lost if the channel quality is severe. Hence even in critical applications where a low-latency protocol is required, Z-MAC is not suitable and performs no better than the proposed protocol. To improve this problem, we can incorporate a message priority or aggregation scheme in the proposed protocol as our future work.

\section{Conclusion}

This paper presents a TDMA-based MAC protocol that incorporates link state dependent scheduling for wireless sensor networks in industrial settings. Because the conditions of the communication channel in industrial environments are harsher than many other situations, existing MAC protocols for WSNs do not perform well under these conditions.

We show that a fully automated and periodically changing environment has a periodic small scale fading effect on radio signals. We integrate object motion in series of snapshots with a known site-specific 3D ray tracer to simulate radio propagation through static and moving objects in two different scenarios of an industrial site, each with different transmitter-receiver placement and different velocities of objects in motion. We find that the placement of nodes in the environment and the velocity of moving objects contribute to the magnitude of signal fading. In the absence of indeterminate sources that cause signal fading, we find that signal fading occurs in approximately periodic bursts.

Our protocol is designed to specifically deal with periodic signal fading in the communication channel. Our approach uses link state dependent scheduling to postpone transmission 
when the channel is predicted to be bad, and only performs transmission/reception when the channel is predicted to be clear. We have demonstrated a simple algorithm used in our link state dependent scheduling approach that can detect patterns of the periodic signal fading. Through extensive simulations, we compared the performance of the proposed protocol with the Z-MAC protocol. We found that our protocol achieved packet delivery ratio of almost $100 \%$ at varying obstruction frequencies and outperformed Z-MAC, both with and without acknowledgement transmissions, when the ideal observation length is used. Where the observation length is insufficient, the performance of our proposed protocol degrades gracefully but is still better than Z-MAC. In addition, our protocol is also energy-efficient because energy is conserved when transmissions are avoided during bad slots.

\section{References}

[1] The network simulator (ns-2). http://www.isi.edu/nsnam/ns/.

[2] M. Arumugam and S. S. Kulkarni. Self-stabilizing deterministic TDMA for sensor networks. In Proceedings of the Fifth European Dependable Computing Conference (EDCC5), April 2005.

[3] J. Aruz and P. Krishnamurthy. Markov modeling of 802.11 channels. In Proceedings of IEEE VTC-Fall, October 2003.

[4] Z. Chen and A. Khokhar. Self organization and energy efficient TDMA MAC protocol by wake up for wireless sensor networks. IEEE SECON 2004, August 2004.

[5] D. R. J. Estes, T. B. Welch, A. A. Sarkady, and H. Whitesel. Shipboard radio frequency propagation measurements for wireless networks. In Military Communications Conference. MILCOM 2001. Communications for Network-Centric Operations: Creating the Information Force. IEEE, pages 247-251, 2001.

[6] D. T. H. Hashemi. Analysis of the rms delay spread of indoor radio propagation channels. IEEE International Conference on Communications, ICC 92, Conference record, SUPERCOMM/ICC 9́2, Discovering a New World of Communications., 2:875-881, 1992.

[7] J. He, A. Verstak, L. T. Watson, C. A. Stinson, N. Ramakrishnan, C. A. Shaffer, T. S. Rappaport, C. R. Anderson, K. Bae, J. Jiang, and W. H. Tranter. S ${ }^{4}$ W : Globally opti- 
mized design of wireless communication systems. Technical Report TR-02-16, Virginia Polytechnic Institute and State University, 2002.

[8] C. T. Inc. Mica2dot datasheet. http://www.xbow.com.

[9] W. L. Lee, A. Datta, and R. C. Oliver. QMAC: A quality of serviceoriented medium access control protocol for data gathering in wireless sensor networks. Technical report, University of Western Australia, August 2005. http://web.csse.uwa.edu.au/research/Technical_Reports?f=86881.

[10] R. C. Levine. Digital switching. Lecture Notes, April 2000. http://www.privateline.com/celpcslevine.pdf.

[11] D. Maniezzo, G. Pau, M. Gerla, G. Mazzini, and K. Yao. T-MAH: A token passing MAC protocol for ad hoc networks. MedHocNet2002, Chia, Sardegna, Italy, September 2002.

[12] V. Phua, A. Datta, and R. Cardell-Oliver. A TDMA-based MAC protocol for industrial wireless sensor network applications using link state dependent scheduling. Technical report, University of Western Australia, February 2006. http://people.csse.uwa.edu.au/valance/TechReport/2006/index.pdf.

[13] D. Puccinelli and M. Haenggi. Multipath fading in wireless sensor networks: Measurements and interpretation. International Wireless Communication and Mobile Computing Conference (IWCMC 2006), 2006.

[14] T. S. Rappaport. Wireless Communications: Principles and Practice. Prentice-Hall PTR, 2nd edition, 2002.

[15] I. Rhee, A. Warrier, M. Aia, and J. Min. Z-MAC: A hybrid MAC for wireless sensor networks. ACM Conference on Embedded Networked Sensor Systems (Sensys), November 2005.

[16] A. A. M. Saleh, J. A. J. Rustako, J. Leonard J. Climini, G. J. Owens, and R. S. Roman. An experimental TDMA indoor radio communications system using slow frequency hopping and coding. IEEE Transactions on Communications, 39(1):152-162, January 1991.

[17] S. Y. Seidel and T. S. Rappaport. Site specific propagation prediction for wireless inbuilding personal communication system design. IEEE Transactions on Vehicular Technology, 43(4):879-891, 1994. 
[18] L. F. W. van Hoesel and P. J. M. Havinga. A lightweight medium access protocol (LMAC) for wireless sensor networks: Reducing preamble transmissions and transceiver state switches. In International Workshop on Networked Sensing Systems (INNS 2004), Tokyo, Japan, June 2004.

[19] A. A. Verstak. Data and computation modeling for scientific problem solving environments. Master's thesis, Virginia Polytechnic Institute and State University, 2002.

[20] A. Willig. Wireless LAN technology for the factory floor. In The Industrial Information Technology Handbook, pages 1-15. 2005.

[21] A. Willig, K. Matheus, and A. Wolisz. Wireless technology in industrial networks. Proceedings of the IEEE, 93(6), June 2005. 2005. 